\title{
Printed Antenna for Satellite Communications
}

\author{
Andrés García-Aguilar, José Manuel Inclán-Alonso, José Manuel Fernández-González, Manuel Sierra-Pérez. \\ Radiation Group. Signals, Systems and Radiocommunications Dept. \\ Universidad Politécnica de Madrid. Avda. Complutense, 30. 28040. Madrid. Spain. \\ andresg@gr.ssr.upm.es,m.sierra.perez@gr.ssr.upm.es,jmfdez@gr.ssr.upm.es, chema@gr.ssr.upm.es.
}

\begin{abstract}
An antenna which has been conceived as a portable system for satellite communications based on the recommendations ITU-R S.580-6 [1] and ITU-R S.465-5 [2] for small antennas, i.e., with a diameter lower than 50 wavelengths, is introduced. It is a planar and a compact structure with a size of $40 \times 40 \times 2 \mathrm{~cm}$. The antenna is formed by an array of 256 printed elements covering a large bandwidth $(14.7 \%)$ at $X$-Band with a VSWR of 1.4:1. The specification includes transmission (Tx) and reception $(\mathrm{Rx})$ bands simultaneously. The printed antenna has a radiation pattern with a $3 \mathrm{~dB}$ beamwidth of $5^{\circ}$, over a $31 \mathrm{dBi}$ gain, and a dual and an interchangeable circular polarization.
\end{abstract}

\section{INTRODUCTION}

This printed antenna for satellite communications has been designed to be a planar, compact, modular, low losses and dual circular polarized antenna, for $\mathrm{Tx}$ and $\mathrm{Rx}$ bands simultaneously (Fig. 1).

Planar antennas are very suitable for personal communications systems due to its capacity to be portable, low profile and low weight. Usually, slots [3] and printed elements [4] are most used as radiating elements.

In this case a square planar array of $16 \times 16$ double stacked microstrip patches, fed by two coaxial probes in order to generate the circular polarization, is used. A hybrid circuit creates the $90^{\circ}$ phase shift to excite two orthogonal modes in the microstrip patch [5]. The array has been divided in 16 square subarrays of $4 \times 4$ elements making possible separate the fabrication of the subarrays from the global distribution network, simplifying the corporative network and getting a modular structure which is suitable in a serial fabrication process. The power distribution network is connected to each subarray with SMP-type coaxial connectors and there are two global inputs/outputs with SMA-type connectors.

The subarray feeding network has two different layers for right-handed circular polarization (RHCP) and left-handed circular polarization (LHCP) in stripline and in microstrip line, respectively. This two printed layers can be manufactured with the microstrip patches at the same time. The global corporative network, which is located under subarrays, is done using a low loss triplate line. Such a kind of feeding network allows keeping a trade-off between the simplicity of exciting the radiating elements using printed circuits and the reduction loss when the distribution network is separated in a structure designed to have low losses.

The antenna works at X-Band with a $14.7 \%$ of relative bandwidth for a VSWR of 1.4:1. Table I summarizes the antenna main characteristics.
TABLE I

ANTENNA SPECIFICATIONS

\begin{tabular}{|c|c|c|}
\hline Parameter & Specification & Units \\
\hline $\begin{array}{c}\text { Working bands } \\
\text { Tx: } \\
\text { Rx: }\end{array}$ & $\begin{array}{c}7.9 \text { to } 8.4 \\
7.25 \text { to } 7.75\end{array}$ & $\begin{array}{l}\mathrm{GHz} \\
\mathrm{GHz}\end{array}$ \\
\hline Polarization & $\begin{array}{c}\text { Dual circular } \\
\text { polarization for } \mathrm{Tx} \\
\text { and } \mathrm{Rx} \text { bands }\end{array}$ & \\
\hline $\mathrm{G} / \mathrm{T}$ & 7 & $\mathrm{~dB} / \mathrm{K}$ \\
\hline PIRE & 32 & $\mathrm{dBW}$ \\
\hline $3 \mathrm{~dB}$ beamwidth & 5 & degrees \\
\hline Maximum gain & 31 & $\mathrm{dBi}$ \\
\hline Efficiency & $>50$ & $\%$ \\
\hline $\begin{array}{c}\text { Isolation } \\
\text { between } \mathrm{Tx} \text { and } \\
\mathrm{Rx}\end{array}$ & $>17$ & $\mathrm{~dB}$ \\
\hline VSWR & $1.4: 1$ & \\
\hline SLL & -16 & $\mathrm{~dB}$ \\
\hline Size & $40 \times 40 \times 2$ & $\mathrm{~cm}$ \\
\hline
\end{tabular}

\section{ARRAY STRUCTURE}

As it has already been commented, the array is formed by $16 \times 16$ stacked patch divided into equal subarrays of $4 \times 4$ elements. The distance among patches is $25 \mathrm{~mm}(\sim 0.65 \lambda)$. Fig. 1 (a) and (b) shows a scheme of the array structure and in Fig. 1(c) can be seen the layers that make up the antenna structure. The bottom layer is the power distribution network in triplate line. There are two triplate line layers and two inputs/outputs in SMA-type connector, one for each polarization. Next, it can be found the subarray network with a layer in stripline for RHCP and other layer in microstrip technology for LHCP. The upper layer is the stacked microstrip patch. The patch layer and the subarray network forms a unique structure which is interconnected to the global distribution network through two SMP-type coaxial connectors, one for each polarization.

The subarray feeding is not the same for each one, but it has symmetry with regarding to the main two antenna axes (Fig. 2), in order to fulfill the mask imposed by the recommendations ITU-R S.580-6 [1] and ITU-R S.465-5 [2]. Fig. 3 shows the simulated radiation pattern for a $16 \times 16$ array in its band upper frequency for the two main planes, compared to the mask aim, with a relative power distribution of $\mathrm{A}=1$, $\mathrm{B}=0.8$ and $\mathrm{C}=0.6$. As can be seen, this array configuration fulfils the radiation pattern specification apart from the distant side lobes. This behaviour is worst in the upper band frequency than in the lower one. The antenna has a $3 \mathrm{~dB}$ 
beamwidth of $5^{\circ}$ in its main lobe and $31 \mathrm{dBi}$ of maximum directivity at broadside.

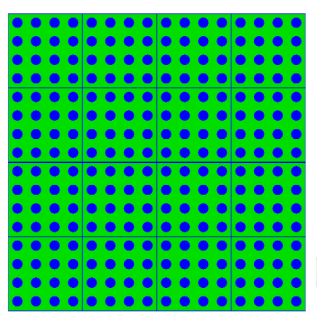

(a)
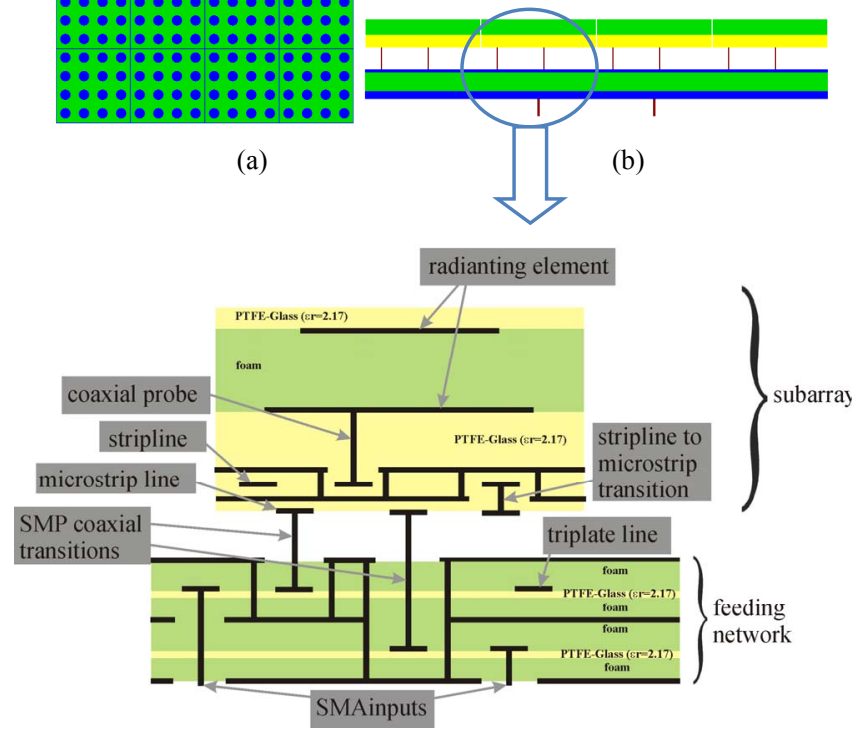

(c)

Fig. 1. The antenna structure. (a) Top view. (b) Side view. (c) Multilayer scheme.

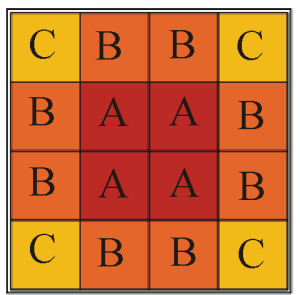

Fig. 2. Subarray feeding distribution.

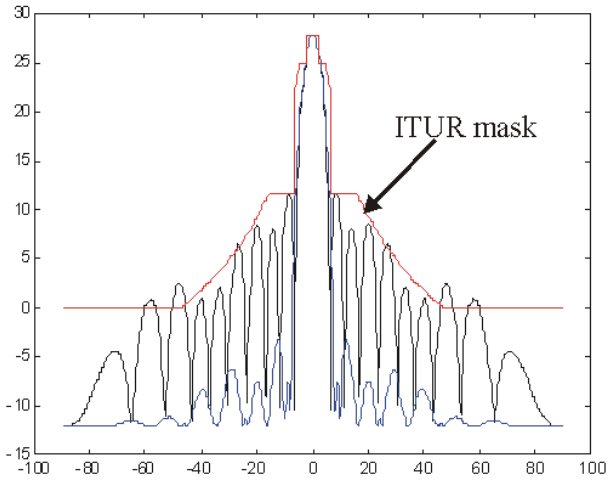

Fig. 3. Radiation pattern for a $16 \times 16$ array in the upper band frequency with the mask aim. With $\mathrm{A}=1, \mathrm{~B}=0.8$ and $\mathrm{C}=0.6$.

\section{RADIATING ELEMENT}

The radiating element is a double stacked microstrip patch excited by two coaxial probes to generate a circular polarization. The lower and the upper patch are printed over PTFE-Glass with a permittivity of 2.17 and are separated by a thick foam layer, as is shown in Fig. 4 together with the structure main dimensions. This element covers the Tx and $\mathrm{Rx}$ bands simultaneously ( $14.7 \%$ of relative bandwidth) with a reflection over $20 \mathrm{~dB}$ and an isolation between probes about $16 \mathrm{~dB}$. Fig. 5 shows the S-parameters of the radiating element simulated by a FDTD software.

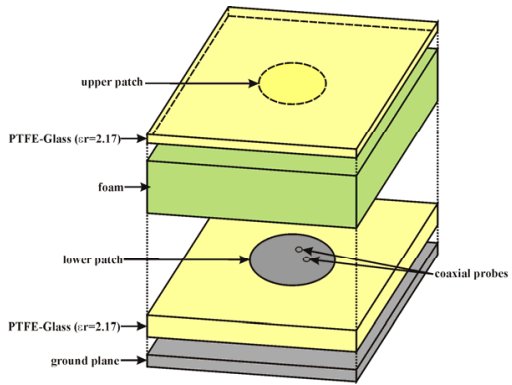

(a)

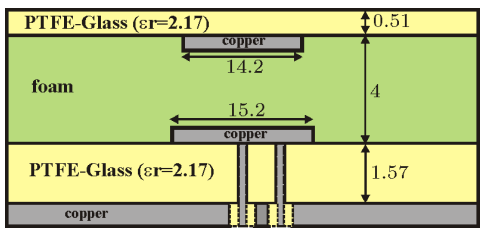

(b)

Fig. 4. Radiating element: circular polarized double stacked microstrip patch excited by two coaxial probes. (a) Perspective view. (b) Side view (dimensions in $\mathrm{mm}$ )

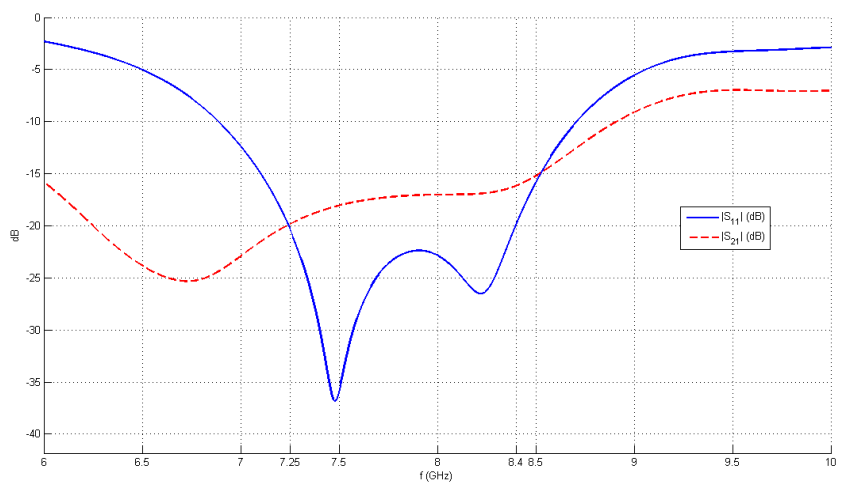

Fig. 5. Radiating element S-parameters.

This configuration fulfills the specifications of radiation pattern and matching impedances, but results regarding copolar/crosspolar $(\mathrm{CP} / \mathrm{XP})$ ratio and isolation are poorer due to a very thick lower substrate which leads to a parasitic radiation and a coupling between coaxial probes. In general, the XP component and the coupling can be reduced combining several elements in the array, like sequential rotation elements [5]. 


\section{SUBARRAY SUBSYSTEM}

The array is divided into subarrays of $4 \times 4$ elements with a distance among elements of $25 \mathrm{~mm}$. The subarray feeding network is implemented in stripline and in microstrip technologies. As the radiating elements and the feeding network formed a unique multilayer board, each subarray board is going to have a size of $9.9 \times 9.9 \times 0.74 \mathrm{~cm}$. Fig. 6 shows a diagram of the subarray multilayer board.

The estimated losses for the feeding network in stripline are about $2.3 \mathrm{~dB} / \mathrm{m}$ and for the microstrip layer is about $3.6 \mathrm{~dB} / \mathrm{m}$, which lead to $0.35 \mathrm{~dB}$ and $0.6 \mathrm{~dB}$, respectively, of total losses.

In order to improve the $\mathrm{CP} / \mathrm{XP}$ ratio a sequential rotation has been established among two by two elements, achieving about $40 \mathrm{~dB}$ of improvement. This configuration has been selected considering the trade-off between simplicity in the subarray feeding network and the increase in polarization purity.

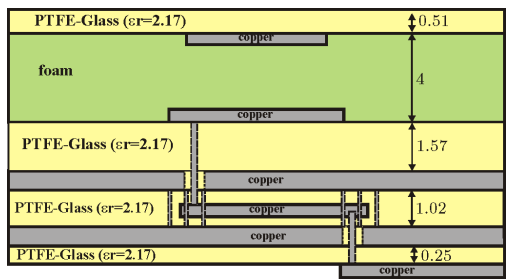

Fig. 6. Subarray multilayer structure (dimensions in $\mathrm{mm}$ ).

\section{A. Vertical transitions between layers}

Vertical transitions, through metalized via holes, allow connection between the different board layers. On the one hand between the microstrip and the stripline layers and on the other hand from the stripline and the lower patch layer. These transitions have to be protected in the stripline layer to avoid unwanted higher-order modes propagation by placing metalized via holes between the ground planes of the stripline. In Fig. 7 is presented the stripline to microstrip line transition and in Fig. 8 is shown the stripline to lower patch transition.

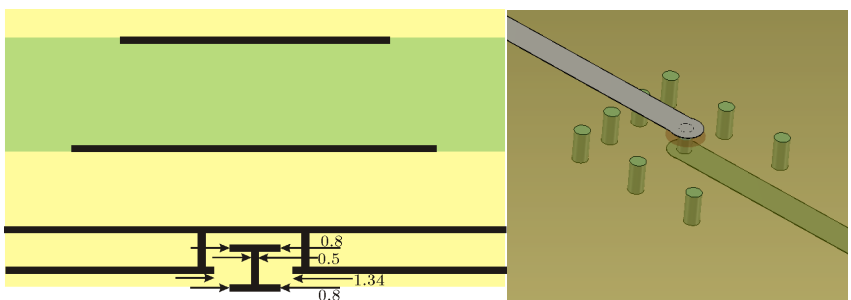

Fig. 7. Stripline to microstrip transition (dimensions in $\mathrm{mm}$ ).
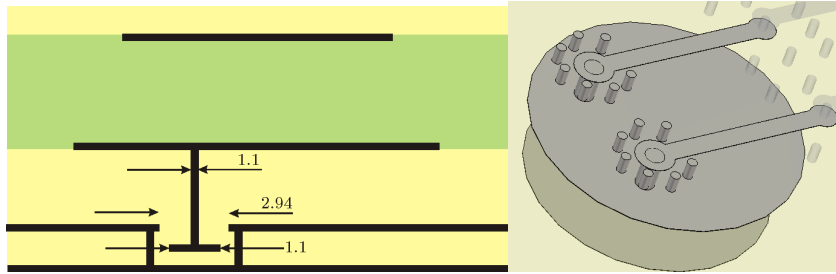

Fig. 8. Stripline to lower patch transition (dimensions in $\mathrm{mm}$ ).

\section{B. Hybrid circuit}

To achieve two orthogonal modes in the circular polarized patch it is necessary to excite probes with a quadrature signal. Moreover, using a $3 \mathrm{~dB}$ hybrid circuit it is possible to work with a RHCP and a LHCP simultaneously with an isolation that will depend on the frequency band and on the matching impedances in the antenna terminals.

A three branches periodic $3 \mathrm{~dB}$ branch-line coupler (BLC) has been chosen in order to cover the working bandwidth [6]. This BLC is located in the stripline layer and its dimensions are shown in Fig. 10(a). It takes up an area of $116 \mathrm{~mm}^{2}$ which is big compared with the radiating element and the subarray subsystem size. Therefore, it is needed to miniaturize this BLC using the equivalence between a $\lambda / 4$ transmission line and a line with an open-ended shunt stub [7], as can be seen in Fig. 9. There are other miniaturization alternatives such as using fractal shapes [8] or interdigitally-coupled lines [9], but this solution has been chosen due to its simplicity and good results.

The miniaturized BLC (from here on the nonminiaturized BLC will be called conventional BLC) dimensions are shown in Fig. 10(b). It has an area of $70 \mathrm{~mm}^{2}$, which means an area reduction of $40 \%$. Fig. 11 shows a comparative between the simulated conventional and miniaturized BLC S-parameters. It is noticed that the miniaturized BLC has the same behavior than the conventional one; even with a wider bandwidth.

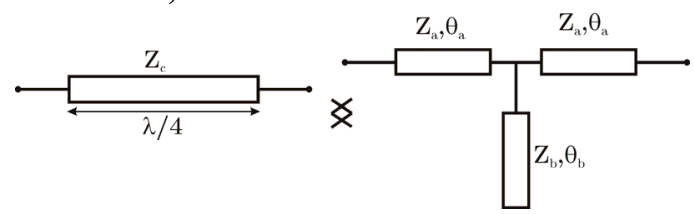

Fig. 9. Equivalence between a $\lambda / 4$ transmission line and a transmission line with an open-ended shunt stub.

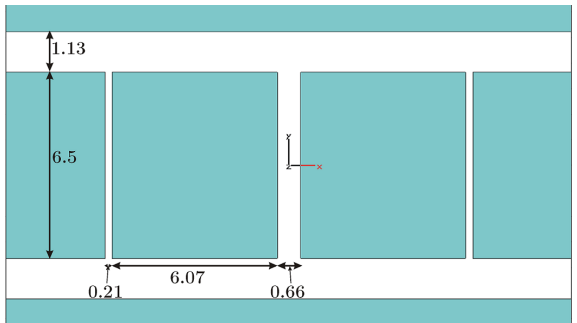

(a)

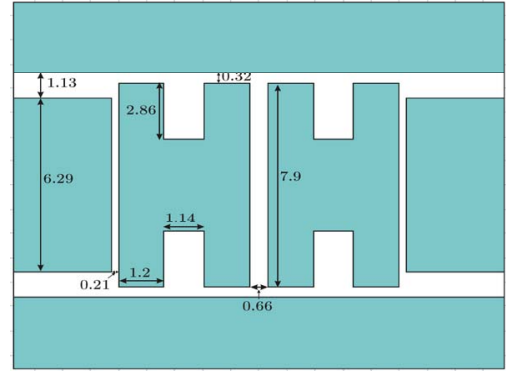

(b)

Fig. 10. Three branches $3 \mathrm{~dB}$ BLC in stripline (dimensions in $\mathrm{mm}$ ). (a) Conventional periodic. (b) Miniaturized version. 


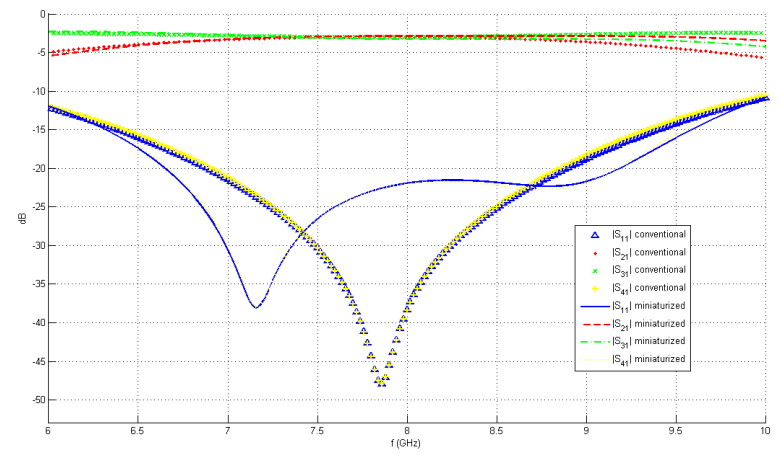

(a)

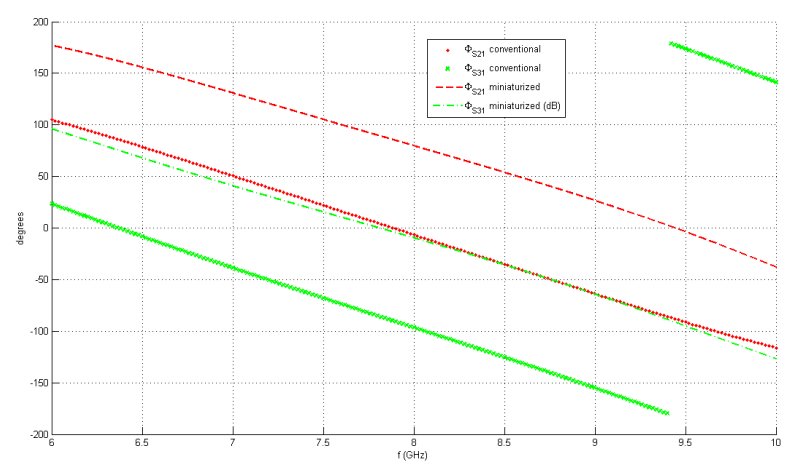

(b)

Fig. 11. S-parameters comparison between a conventional and a miniaturized 3dB BLC: (a) Absolute value. (b) $90^{\circ}$ phase shift behavior.

\section{Subarray feeding network}

Although both polarizations have their subarray feeding network inputs in the microstrip layer through two SMP-type connectors, the subarray feeding network is formed by two layers in stripline and in microstrip for RHCP and LHCP, respectively. A parallel structure has been chosen as is presented in Fig. 12, where the power dividers distribute the signal between every branch. The line lengths have to be designed taking into account the sequential rotation among two by two elements and the desired polarization.

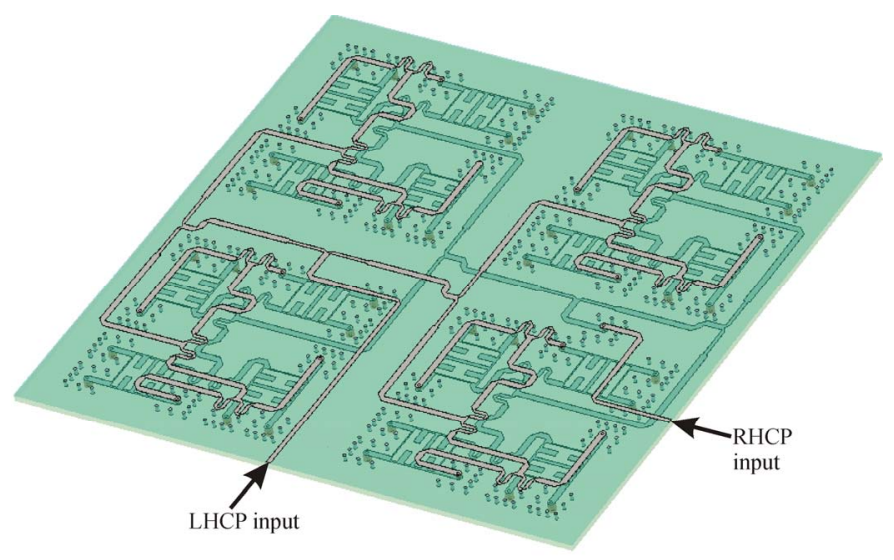

Fig. 12. Subarray feeding network in stripline and microstrip technologies.

\section{GLOBAL POWER DISTRIBUTION NETWORK}

The power distribution network, that takes the signal to each subarray and that is located under the subarray subsystems, is implemented using a low losses triplate line configuration. There is a layer for each polarization to guarantee the polarization isolation. Its multilayer configuration is shown in Fig. 13.

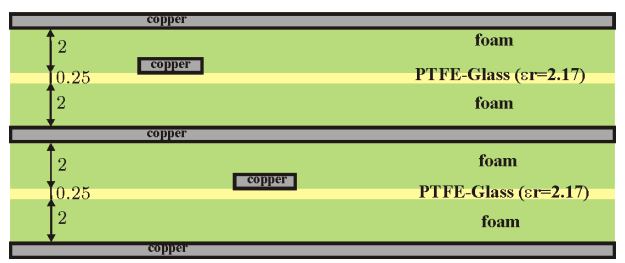

Fig. 13. Global power distribution network multilayer structure (dimensions in $\mathrm{mm}$ ).

To fulfill the radiation pattern of Fig. 3 the amplitude distribution must have the configuration of Fig. 2, with $A=1$, $\mathrm{B}=0.8$ and $\mathrm{C}=0.6$, and $\mathrm{a}$ uniform phase distribution. This power distribution is achieved using power dividers with unbalanced impedance matching.

In the transition between the triplate line and a coaxial connector it is excited a TE mode which can be avoided surrounding the transition with a metallic piece that works as a short-circuited waveguide. Such a configuration can be seen in Fig. 14 (a) and (b). There are two kinds of transitions from a triplate line to a coaxial connector. One is the interconnection between the triplate and the subarrays through SMP-type connectors, whose dimensions can be seen in Fig. 14(c). And the other is the transition between the triplate and the two general inputs/outputs with SMA-type connectors, which is shown in Fig. 14(d) with its size.

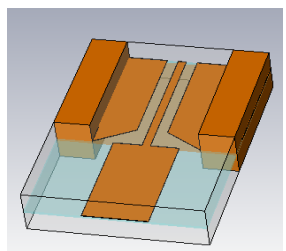

(a)

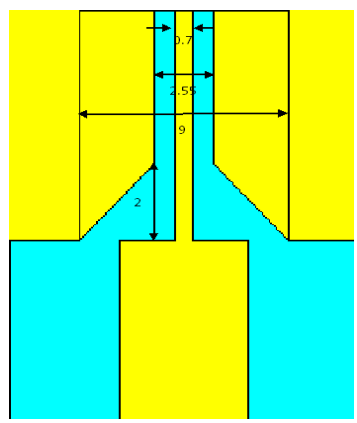

(c)

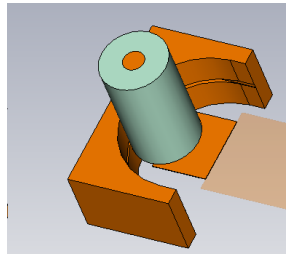

(b)

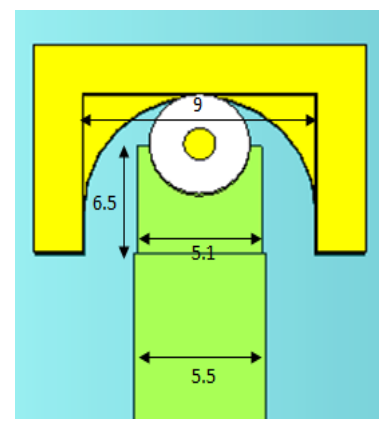

(d)
Fig. 14. Transition from triplate line to (a) SMP-type coaxial connector and (b) to SMA-type coaxial connector. (c) Triplate to SMP dimensions (mm). (d) Triplate to SMA dimensions ( $\mathrm{mm})$. 
Fig. 15 shows the global corporative network for one polarization and in Fig. 16 can be seen the S-parameters simulated with a FDTD software, where is noticed the different relative power amplitude in each subarray. As can be seen reflection coefficient is over $20 \mathrm{~dB}$ in the all frequency band.

The estimated losses in the triplate line are about $0.5 \mathrm{~dB} / \mathrm{m}$ which leads to $0.2 \mathrm{~dB}$ of total losses in the complete network.

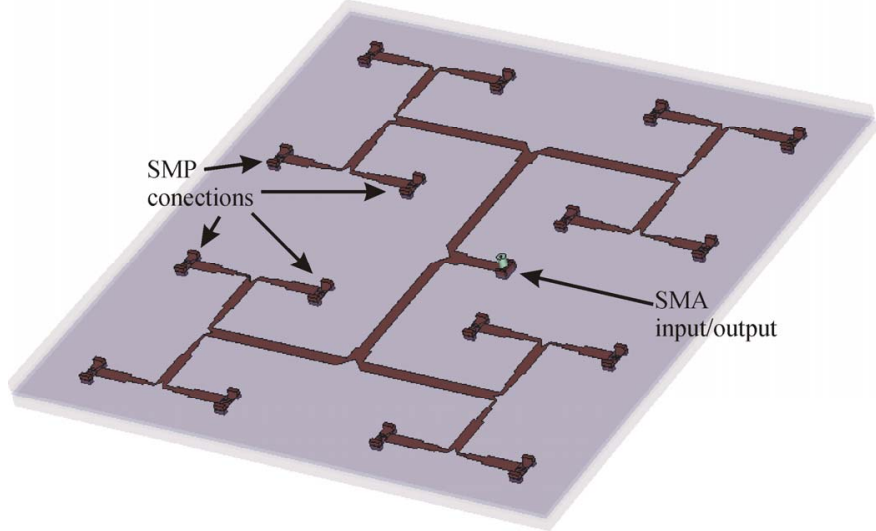

Fig. 15. Global power distribution network in triplate line for one layer.

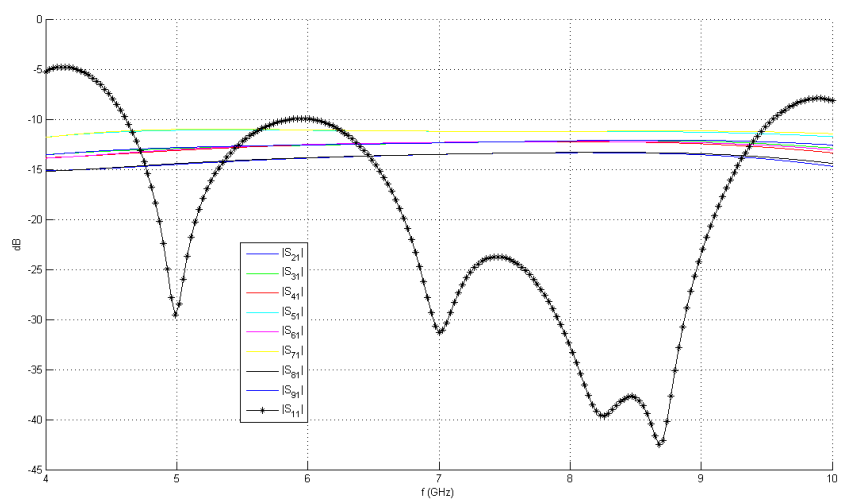

Fig. 16. Power distribution network S-parameters.

\section{CONCLUSIONS}

The complete design of a portable printed antenna for satellite communications at X-Band has been introduced. This antenna has been conceived as a planar, compact, modular, low losses and dual circular polarized antenna for Tx and Rx bands simultaneously. Its size is of $40 \times 40 \times 2 \mathrm{~cm}$ and covers a $14.7 \%$ of bandwidth with a VSWR of $1.4: 1$. The antenna has a radiation pattern with $5^{\circ}$ of $3 \mathrm{~dB}$ beamwidth in its main lobe and $31 \mathrm{dBi}$ of maximum gain.

The array has been divided in 16 square subarrays of $4 \times 4$ elements which are double stacked microstrip patches. Each subarray has a two-layered feeding network, in stripline and in microstrip line, one for each polarization (RHCP and LHCP) to avoid couplings inside network. A miniaturized branch-line coupler has been designed in order to reduce couplings and to simplify the subarray feeding network. The sequential rotation elements technique has been imposed between two by two patches to improve the polarization purity of the antenna. The complete subarray can be fabricated as a unique multilayer board.
Subarrays are fed by a global power feeding network implemented in a low loss triplate line. The corporative network distributes the correct amplitude, with uniform phase, to each subarray in order to accomplish the radiation pattern specifications.

Simulation results are very promising. The first prototype and some measurements results will be presented at the EuCAP 2010 meeting.

\section{ACKNOWLEDGMENT}

Simulations done in this work have been realized using CST Microwave Studio Suite 2009 under a cooperation agreement between Computer Simulation Technology (CST) and Universidad Politécnica de Madrid. The project is leadered by the Universidad Politécnica de Madrid and Antenas Moyano S.L. and the support of the Spanish Education Ministry (Comisión Interministerial de Ciencia y Tecnología) under reference TEC2008-06736/TEC, two Spanish Goberment Grant (FPU and FPI under reference BES-2009-021462) and the help of European Cost Action IC0603 Assist. NY substrate used in the prototypes was kindly given by NELTEC S.A.

\section{REFERENCES}

[1] Radiation diagrams for use as design objectives for antennas of earth stations operating with geostationary satellites, Jan. 2004, ITU-R Recommendation S.580-6.

[2] Reference earth-station radiation pattern for use in coordination and interference assessment in the frequency range from 2 to about $30 \mathrm{GHz}$, Apr. 1993, ITU-R Recommendation S.465-5.

[3] M. Sierra-Castañer, M. Vera-Isasa, M. Sierra-Pérez, J.L. Fernández-Jambrina, "Double-Beam Parallel Plate Slot Antenna", IEEE Transactions on Antennas and Propagation, vol. 53, no. 3, pp. 977-984, Mar. 2005.

[4] A. Stark, et al., "SANTANA: Advanced electronically steerable antennas at Ka-Band", 3rd European Conference on Antennas and Propagation, EuCAP 2009, Berlin, pp. 471-478, Mar. 2009.

[5] R. Garg, P.Bhartia, I. Bahl, A. Ittipiboon, Microstrip Antennas Design Handbook, Artech House, 2001.

[6] G. Matthaei, L. Young, E.M.T. Jones, Microwave filters, impedance-matching networks, and coupling structures, Artech House, 1980.

[7] Ching-Wen Tang, Ming-Guang Chen, "Synthesizing Microstrip Branch-Line Couplers With Predetermined Compact Size and Bandwidth", IEEE Trans on Microwave Theory and Techniques, vol. 55, no. 9, pp. 1926-1934, Sep. 2007.

[8] Wen-Ling Chen, Guang-Ming Wang, "Design of Novel Miniaturized Fractal-Shaped Branch-Line Couplers", AsiaPacific Microwave Conference, APMC 2007, 11-14 Dec. 2007, pp. 1-3.

[9] Zhiyang Liu, R.M. Weikle, "A Compact Quadrature Coupler Based on Coupled Artificial Transmission Lines", IEEE Microwave and Wireless Components Letters, vol. 15, no. 12, pp. 889-891, Dec. 2005. 